\title{
新型冠状病毒肺炎疫情下综合医院发热门诊环境监测与 感染控制
}

陆依然 ${ }^{1,2 \dagger}$, 李伊凡 ${ }^{1,2 \dagger}$, 林明贵 $3^{*}$, 刘荔 ${ }^{1,2^{*}}$, 林波荣 ${ }^{1,2}$, 周浩 ${ }^{1,2}$, 林金兰 ${ }^{3}$, 徐沪济 $^{3}$

1. 清华大学建筑学院, 北京 100084 ;

2. 清华大学生态规划与绿色建筑教育部重点实验室, 北京 100084;

3. 清华大学附属北京清华长庚医院感染科, 北京 102218

$\dagger$ 同等贡献

*联系人, E-mail: linminggui309301@126.com; liuli_archi@tsinghua.edu.cn

2020-08-03 收稿, 2020-09-23 修回, 2020-09-24 接受, 2020-09-25 网络版发表

国家重点研发计划(2020YFC0842500)、国家自然科学基金(52041602)和清华大学附属北京清华长庚医院抗疫专项(12020Z1003)资助

摘要 新型冠状病毒肺炎疫情暴发初期, 社会性恐慌导致感染新型冠状病毒的患者和有类似症状的患者涌向医院, 尤其是发热门诊. 此外, 还有部分已感染新型冠状病毒的患者到医院其他科室就诊. 疫情初期，由于医护人员个人 防护物资储备不足及防护意识不到位, 部分医护人员被感染, 因此, 必须严格控制医院内环境, 减少医院内近距离 人际接触频率, 防止新型冠状病毒通过飞沫、接触等途径传播. 本研究以清华大学附属北京清华长庚医院为例, 基 于二氧化碳 $\left(\mathrm{CO}_{2}\right)$ 浓度的实时监测，建立了评估医院通风稀释情况、预警发热门诊内患者间传播风险的环境控制 方法。根据发热门诊候诊区走廊、护理站和医生诊室在室人数限值及规定新风量，计算出上述区域内二氧化碳浓 度理论上限值. 环境监测期间, 医护人员均采取二级防护, 未有医患间感染发生; 共接诊5 名新型冠状病毒肺炎确诊 患者, 确诊患者停留在发热门诊期间, 就诊患者佩戴口罩, 候诊区走廊、护理站和医生诊室二氧化碳浓度均低于理 论上限值且分别控制在 $609 、 654$ 和 $711 \mathrm{ppm}\left(1 \mathrm{ppm}=10^{-6} \mathrm{~mol} / \mathrm{mol}\right)$ 以下, 患者间未发生交叉感染. 该经验与方法可供 同类型综合医院发热门诊设计与改造参考.

关键词 新型冠状病毒肺炎, 医院环境, 监测预警, 感染防控

2019年底，新型冠状病毒肺炎(简称“新冠肺炎”)疫 情突然暴发. 经过半年的同心抗疫, 现阶段我国新冠肺 炎疫情基本得到控制，但是疫情仍在全球蔓延. 截至 2020 年7月 29 日, 全球已确诊超过 1652 万例, 其中 65 万 例确诊患者死亡, 增长速度未见放缓趋势, 第一波感染 浪潮尚未结束(https://covid19.who.int/).

中国国家卫生健康委员会(National Health Commission of the People's Republic of China, NHC)、世 界卫生组织(World Health Organization, WHO)和美国
疾病控制与预防中心(Centers for Disease Control and Prevention, CDC)等机构对新型冠状病毒(简称“新冠病 毒”)主要传播途径的看法一致, 但对空气传播、排泄物 接触及气溶胶吸人等途径是否存在、存在的条件有较 大分歧. 各机构对新冠病毒传播途径的界定见表 1 (https://www.who.int/news-room/commentaries/detail/ modes-of-transmission-of-virus-causing-covid-19-implications-for-ipc-precaution-recommendations, http://www. nhc.gov.cn/yzygj/s7653p/202003/46c9294a7dfe4-

引用格式: 陆依然, 李伊凡, 林明贵, 等. 新型冠状病毒肺炎疫情下综合医院发热门诊环境监测与感染控制. 科学通报, 2021, 66: 475-485 Lu Y R, Li Y F, Lin M G, et al. Environmental monitoring and infection control of fever clinics in general hospitals during COVID-19 pandemic (in Chinese). Chin Sci Bull, 2021, 66: 475-485, doi: 10.1360/TB-2020-0969 
表 1 中国国家卫生健康委员会、世界卫生组织、美国疾病控制与预防中心对新冠病毒传播途径的界定

Table 1 Definition of COVID-19 transmission routes by National Health Commission of China, World Health Organization and the US Centers for Disease Control and Prevention

\begin{tabular}{|c|c|c|c|c|c|c|}
\hline \multirow{2}{*}{ 机构 } & \multirow{2}{*}{ 飞沫传播 } & \multicolumn{2}{|c|}{ 接触传播 } & \multirow{2}{*}{$\begin{array}{c}\text { 空气/ } \\
\text { 气溶胶传播 }\end{array}$} & \multicolumn{2}{|c|}{ 粪便、尿液 } \\
\hline & & 直接接触 & 间接接触 & & 粪-接触 & 粪-呼吸道 \\
\hline 中国国家卫生健康委员会 & 确定 & 确定 & 确定 & 存在 $^{\text {a) }}$ & 可能存在 ${ }^{\mathrm{c}}$ & 可能存在 ${ }^{c)}$ \\
\hline 世界卫生组织 & 确定 & 确定 & 确定 & 医院内存在 ${ }^{b)}$ & & \\
\hline 美国疾病控制与预防中心 & 确定 & 确定 & 部分情况 & & & \\
\hline
\end{tabular}

a) 相对封闭空间长时间暴露于高浓度气溶胶情况下存在气溶胶传播的可能; b) 气溶胶传播仅存在于医院内插詙管、气切等产生人源气 溶胶的医疗操作; c) 在粪便及尿中可分离到新冠病毒, 应注意粪便及尿对环境污染造成气溶胶传播或接触传播

cef80dc7f5912eb1989.shtml, https://www.cdc.gov/coronavirus/2019-ncov/prevent-getting-sick/how-covidspreads.html).

新冠肺炎疫情暴发初期与季节性流感时间段重合, 由于感染新冠病毒和流感病毒后都会出现乏力、发热 和咳嗽等症状，新冠肺炎患者和有疑似症状的未感染 者均前往医院就诊，造成医院内尤其是发热门诊人流 持续过度密集, 人流密度可达 $1 \sim 2$ 人 $/ \mathrm{m}^{2}$, 超出了医院环 境设计承载量, 难以保持人际间距大于 $1.5 \mathrm{~m}^{[1,2]}$. 感染 患者与未感染患者长时间排队、近距离共处，存在交 叉感染风险. 新冠肺炎疫情下, 发热门诊就诊人群中新 冠肺炎潜在患者的存在概率高于其他科室，同时间其 他就诊患者的免疫力可能低于健康人群，因而可能更 加易感. 医护人员在诊疗及看护过程中需要近距离、 长时间密切接触患者, 时刻面临被感染的风险. 截至 2020 年 2 月 11 日，我国共有 3019 名医护人员感染，其中 1716 名为核酸确诊病例 ${ }^{[3]}$. 医院诊疗环境的安全十分 重要, 迫切需要更加量化的评估方式和更加有效的控 制手段，以降低新冠肺炎等新发、再发传染病的传播 风险.

在无法识别传染源的前提下，现有的医院环境控 制措施主要分三类: 一是控制就诊患者数量, 增加人际 间距, 减少医院内聚集行为; 二是利用新风系统、高效 过滤系统和空气消毒系统, 稀释、净化医院内空气; 三 是定时表面消毒, 降低间接接触传播风险. 医院内环境 控制必须以实际环境为基础, 明确环境控制手段. 因此, 通过实时监测掌握量化的医院内环境信息至关重要. 现有研究中，在收治新冠肺炎患者的医院中进行环境 监测, 以新冠病毒的表面采样和空气采样为主. 采集到 的样本必须严格按照生物安全要求处理及包装，再通 过核酸检测技术进行处理, 测序相关操作须在生物安
全二级以上的实验室中进行, 检测耗时且流程复杂. 此 类环境监测方法成本较高, 具有滞后性, 无法实时反映 医院内环境污染情况，因而无法及时预警医院内环境 控制失效情况，无法向可能出现院感风险的区域发出 警示信号. 此外, 即使能够耦合实时空气采样及高通量 基因测序技术, 短期内也难以缩短测序时间, 实现新冠 病毒等新发强传染性病原体环境载量的实时监测. 总 之, 新发、突发传染病暴发期间, 亟须寻求更为普适、 实时、便捷的环境监测方法来表征医院环境的通风控 制和环境污染情况，并进一步识别医院内感染风险事 件, 以针对性地提出有效控制方案.

环境监测需求的迫切性促使我们将目光转向现已 成熟且被验证可行可靠的环境监测系统. 通过自主研 发的环境参数集成监测系统，末端监测设备通电即可 以实现对环境温/湿度、二氧化碳浓度、 $\mathrm{PM}_{2.5}$ 浓度和 照度的高时间精度实时监测, 数据可实时显示并上传 至云端进行分析.

我们于 2020年1月20日建立研究方案, 确定使用室 内环境监测仪测量医院室内环境参数，间接表征医院 室内环境承载能力及院感风险，并于2020年1月30日起 在清华大学附属北京清华长庚医院(下称“长庚医院”) 进行方案验证性研究. 我们在发热门诊、隔离病房、 门诊大厅、急诊等功能不同、院感风险各异的区域布 置了室内环境参数监测设备, 在疫情暴发期间持续监 测医院室内环境, 最终拓展为延续至今的常态化监测. 长庚医院为综合性医院，其发热门诊自2020年1月14日 起, 先后接诊新冠肺炎确诊患者 13 名 ${ }^{[4]}$, 从1月 30 日开始 监测后，接诊了5 名确诊患者，期间未发生医护或其他 就诊患者感染. 据流行病学调查确认, 患者间无院内相 互暴露历史. 本研究旨在通过分析长庚医院环境参数, 明确新冠肺炎疫情期间发热门诊的环境特征，结合医 
院对患者行为控制手段，建立平疫结合的发热门诊院 感控制方法.

\section{1 医院室内环境监测与预警}

新冠病毒主要通过患者呼出飞沫和飞沫核传播. 在医院内发现通风不良、缺乏有效稀释的区域时，必 须予以特别关注. 尽管目前无法通过直接监测医院环 境中的病毒载量来判断传播风险，但针对发热门诊这 类感染患者出现可能较高的区域，可通过二氧化碳浓 度示踪发热门诊内所有人员呼出气的通风稀释情况, 间接表征气溶胶传播风险水平. 疫情暴发期内, 可将监 测范围拓展至全院，实时监测、识别院感暴发的风险 区域，发出预警提示，并提出增加新风稀释、过滤净 化、降低人流等防控措施.

\section{1 长庚医院及其发热门诊基本情况}

2020年春节期间，长庚医院的急诊、儿科门诊、 发热门诊持续开诊. 为便于人员流线管理, 减少不必要 的感染, 院方建议非紧急患者暂时不来院就诊, 且除急 诊和发热门诊外，取消现场挂号，全面采用预约挂号， 并建议患者在预约时间段分散前往就诊; 要求有发热 症状、就诊前两周内去过武汉地区或其他本地病例持 续传播地区、就诊前两周内明确接触过武汉地区或其 他本地病例持续传播地区居住者，以及身边有多人发 热的患者，主动前往发热门诊接受笁查. 另外，院方在 各人口处安排预检分检台，对来院人员进行体温检测 和流行病学调查, 并要求患者及家属在就诊时佩戴口 罩, 限制陪同看诊家属人数为 1 人.

长庚医院发热门诊为新冠肺炎定点发热门诊，承 担新冠肺炎患者的诊治和高风险患者的篮查工作，24 小时接诊且提供现场挂号，其中普通患者和新冠肺炎 患者混杂, 若同时就诊人数较多, 则有聚集性感染的风 险, 因此需要重点关注发热门诊的诊疗环境承载力. 发 热门诊的诊疗环境承载力受诊疗各环节功能空间容 量、人均诊疗时长及通风控制情况限制. 为防止院感, 发热门诊医护人员一律采取二级防护，且对院区进行 定时消杀.

长庚医院的暖通空调设计 ${ }^{[5]}$ 依据《采暖通风与空 调设计规范(GB50019-2003)》《民用建筑采暖通风与 空气调节设计规范(GB50736-2012)》《综合医院建筑 设计规范(JGJ49-88)》《医院洁净手术部建筑技术规范 (GB50333-2002)》《公共建筑节能设计标准(DB11/687-
2009)》等. 此次新冠肺炎疫情期间, 由于不明确通风空 调系统对新冠病毒传播的作用，发热门诊采用自然 通风.

\section{2 知院室内环境监测区域}

室内环境监测仪主要布置在医院内病患和医护人 员的聚集停留区. 所选取的 32 个测点覆盖了长庚医院 门诊部、发热门诊、办公楼及综合楼四幢独立楼栋中 的诊室、留观监护病房、CT(Computed Tomography) 室、隔离病房等可能出现新冠病毒携带者的就诊及候 诊空间和人员密集的办公区(图1)，测点覆盖面积共计 约 $4000 \mathrm{~m}^{2}$. 设备布置在墙面或桌面上，距离地面高度 $0.8 \sim 1.5 \mathrm{~m}$ 不等.

\section{3 知院室内环境监测参数}

在长庚医院内布置的室内环境监测设备可以实现 对医院内环境温度、相对湿度、二氧化碳浓度、 $\mathrm{PM}_{2.5}$ 浓度和照度 5 个环境参数的实时高时间精度测量, 环境 参数测量时间间隔为 $5 \mathrm{~min}$.

长庚医院室内环境温/湿度对新冠病毒的影响主要 体现在两个方面：一是影响物表及空气中新冠病毒的 活性，二是影响人体呼出可能含有新冠病毒飞沫的蒸 发与扩散. 已有研究表明, 在温度为 $21 \sim 23^{\circ} \mathrm{C}$ 、相对湿 度为 $40 \%$ 的环境中，新冠病毒可在气溶胶中存活 $3 \mathrm{hW}$ 上，在塑料和不锈钢表面则可存活 $3 \mathrm{~d}^{\circ}$ 上 $^{[6]}$. 从室内 温/湿度对人体呼出飞沫蒸发速度的影响来看，若室内 温/湿度利于携带目标病原微生物的飞沫蒸发，则会有 更多携带病毒的飞沫在沉降至物表前蒸发为飞沫核, 在空气中长时间悬浮, 可能增加院感风险. 对呼出飞沫 的实验研究和数值模拟结果显示，人体通过咳嗽和打 喷德等呼吸活动释放的飞沫在高温干燥环境中更易蒸 发形成飞沫核 ${ }^{[7]}$. 但是, 该过程中病毒活性如何变化, 目 前尚未有定论, 需通过实验获取更多信息后综合判断.

医院室内环境中的二氧化碳来源主要为室外大气 和室内人员.一般情况下, 当忽略汽车尾气排放、焚烧 炉等强二氧化碳源影响时，室外源对室内二氧化碳浓 度的贡献稳定. 我们认为, 院内二氧化碳浓度的波动由 室内人员呼出造成，可以用二氧化碳浓度表征当前监 测时刻人均新风量是否充足及稀释是否充分. 室内二 氧化碳浓度越接近室外浓度，表示通风稀释情况越好， 室内空气越新鲜，当室内存在新冠肺炎感染患者时，同 室人员通过气溶胶和飞沫被感染的风险越低. 

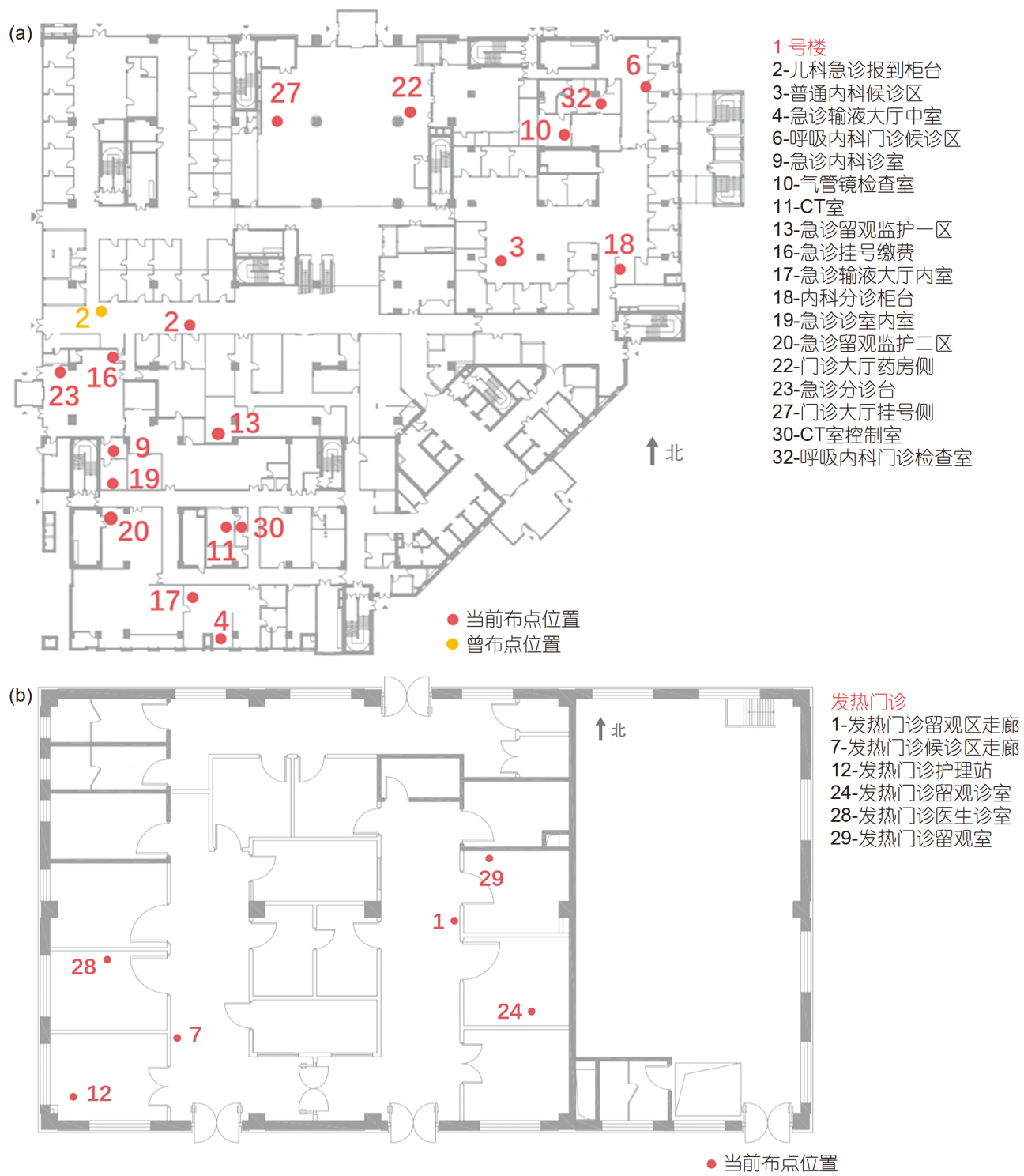

图 1 (网络版彩色)门诊部(a)和发热门诊(b)室内环境监测仪布点详图

Figure 1 (Color online) Detailed layout of indoor environment monitors at the outpatient department (a) and fever clinic (b)

医院 $\mathrm{PM}_{2.5}$ 室内浓度与室外浓度之比值 $(\mathrm{I} / \mathrm{O})$ 大于或 等于1时，表明颗粒物室内源占主导. 医院通过性环境 (候诊环境)中, $\mathrm{PM}_{2.5}$ 主要来源为室内污染源. 人员活动 如衣物摩擦、地面灰尘再悬浮、皮屑脱落以及呼吸、 讲话、咳嗽、打喷德等活动均有颗粒物产生. 此外, 医 院诊疗环境中还存在大量因医疗操作产生的气溶胶. 虽然空气中 $\mathrm{PM}_{2.5}$ 并不一定携带致病微生物, 但是研究 表明, 医院环境中颗粒物计数浓度与生物气溶胶浓度 呈正相关 ${ }^{[8]}$. 对医院 $\mathrm{PM}_{2.5}$ 室内浓度进行实时监测时, $\mathrm{PM}_{2.5}$ 浓度上升, 说明可能室内由于通风失效出现了颗 粒物的累积, 或者室内出现了现有通风量难以满足其
稀释要求的强颗粒物散发源, 需要及时检查通风系统, 并结合监控视频、患者护理记录等对室内 $\mathrm{PM}_{2.5}$ 浓度骤 然升高进行溯源、院感风险识别.

\section{4 发热门诊聚集性院内感染风险预警指标}

长庚医院发热门诊留观区接纳就诊患者少, 因此 本文着重关注患者聚集度高的医生诊室、护理站和候 诊区走廊. 疫情期间, 发热门诊医生诊室和护理站通过 开启外窗进行自然通风，与候诊区走廊相连通的内门 常开. 为控制人流, 候诊区走廊的外门常闭(图2). 自然 通风作为一种被动的通风措施，有时能达到远高于机 
(a)

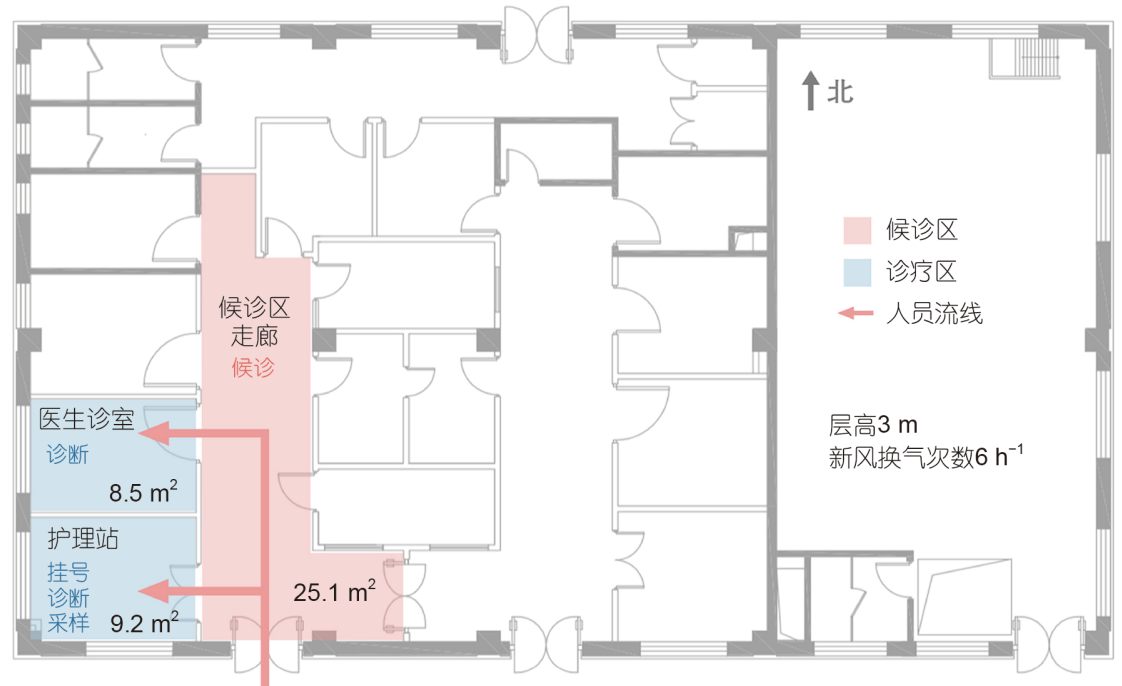

(b)
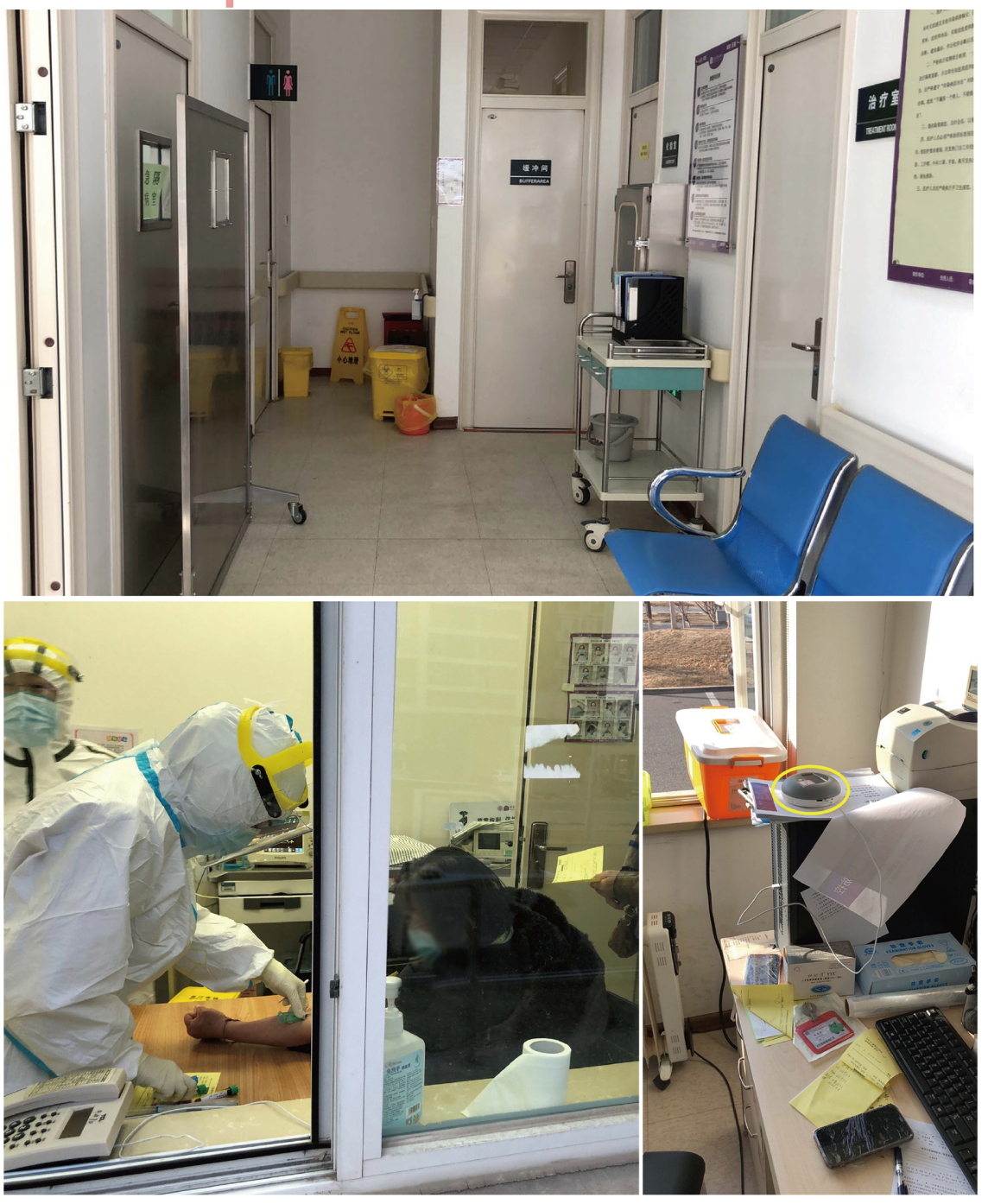

图 2 (网络版彩色)发热门诊测点情况. (a) 发热门诊就诊流程图和测点位置图; (b) 发热门诊候诊区走廊; (c) 位于发热门诊护理站的环境监测仪 Figure 2 (Color online) Environmental monitoring at the fever clinic. (a) Diagram of the fever clinic and detailed layouts of the sampling locations; (b) the waiting area including the corridor; (c) the monitor at the nursing station 
械通风的风量, 以满足传染病房的通风稀释需求. 但是, 自然通风风量受外门外窗的开度、室内外温差、室外 风场等诸多因素的影响，一天之内、不同季节之间会 有较大波动, 与机械通风相比具有较大的不确定性. 发 热门诊院感风险较高, 需要量化确定其自然通风稀释 情况.

发热门诊室内空气中二氧化碳浓度的波动主要受 室内人员的影响. 室内人员呼出的二氧化碳作为自然 示踪气体，通过对室内外二氧化碳浓度以及室内人员 数量、活动强度的持续监测和记录，可以计算室内实 际换气次数、通风量等通风控制参数 ${ }^{[9]}$. 发热门诊的 功能决定了就诊人员中可能存在新冠肺炎患者. 以最 不利情况估计, 即假设就诊患者均为新冠肺炎患者, 则 室内二氧化碳浓度与室外的差值可以近似表征患者呼 出气溶胶造成的感染风险.

在发热门诊的诊疗空间，即医生诊室、护理站以 及候诊区走廊这 3 个测点各布置一套环境监测设备, 获 得了高时间精度的二氧化碳浓度数据. 通过设定室内 人数限值及新风量标准来计算室内二氧化碳浓度的理 论控制上限(表 2$)$, 并将室内二氧化碳浓度实测数据与 该理论限值比较, 以此判断室内通风稀释情况是否满 足通风控制要求.

诊疗空间和候诊空间的人流控制方法及空间使用 模式有所差异，需采用不同的室内人数限值选取方法. 诊疗空间中医患间产生确定的近距离暴露, 通过医护 二级防护及严格限制室内人数降低院感风险, 每个房 间中除 2 名值班医生以外，仅允许最多 2 名患者同时就 诊，因此诊室及护理站室内人数控制在 $2 \sim 4$ 人，取室内 人数上限 $N_{\mathrm{u}}$ 为 4 人. 候诊区走廊中无医护, 使用人员仅 为患者及其家属, 空间内人数变化范围大, 可能无人候 诊, 也可能有大量患者排队候诊, 人数波动与患者抵达 发热门诊的时间分布、每个患者的就诊时长、家属陪 护情况等有关. 候诊区院感风险主要来自就诊患者聚 集导致的密切接触. 以平均人际间距 $1.5 \mathrm{~m}^{[2]}$ 为密切接 触界限, 假设人员在室内均匀分布, 计算候诊区内的人 员控制数量:

$N_{\mathrm{u}}=\operatorname{Int}\left[A / d^{2}\right]$,

其中, $N_{\mathrm{u}}$ 为测点区域内控制人数上限, 人; $A$ 为测点区域 面积, $\mathrm{m}^{2} ; d$ 为平均人际间距控制值, 取 $1.5 \mathrm{~m}$; Int[ ] 函数 表示计算结果向下取整. 代人长庚医院发热门诊候诊 区走廊面积 $25.1 \mathrm{~m}^{2}$ ，计算得到区域控制人数上限为 11

\section{表 2 发热门诊通风控制限值}

Table 2 Ventilation control limits at the fever clinic

\begin{tabular}{cccc}
\hline 测点区域 & $\begin{array}{c}\text { 控制人数 } \\
\text { 上限 } N_{\mathrm{u}} \text { (人) }\end{array}$ & $\begin{array}{c}\text { 人员密度控制 } \\
\text { 范围 } x\left(\text { 人 } / \mathrm{m}^{2}\right)\end{array}$ & $\begin{array}{c}\text { 二氧化碳浓度 } \\
\text { 理论控制限值 } \\
C_{\mathrm{u}}(\mathrm{ppm})\end{array}$ \\
\hline 候诊区走廊 & 11 & $0 \sim 0.44$ & 867 \\
护理站 & 4 & $0.22 \sim 0.44$ & 864 \\
医生诊室 & 4 & $0.24 \sim 0.47$ & 902 \\
\hline
\end{tabular}

人. 若室内人数超过该限值, 则必然出现人际间距小于 $1.5 \mathrm{~m}$ 的密切接触情况.

为排除各测点区域空间面积差异带来的影响, 可 将室内人员数量换算为人员密度, 以便进行院内测点 空间之间的比较, 并可推广至不同医院之间的比较. 将 各测点区域室内人数控制限值转化为室内人员密度控 制限值, 得到室内人员密度控制范围:

$x=N / A$,

其中, $x$ 为测点区域内控制人员密度, 人 $/ \mathrm{m}^{2} ; N$ 为测点区 域内控制人数, 人; $A$ 为测点区域面积, $\mathrm{m}^{2}$. 本研究中, 对 候诊区走廊取控制人数上限 11 人，计算得到人员密度 控制范围为 $0 \sim 0.44$ 人 $/ \mathrm{m}^{2}$. 根据就诊空间人数波动范围 2 4人, 计算得到护理站和诊室人员密度正常范围分别 为 $0.22 \sim 0.44$ 和 $0.24 \sim 0.47$ 人 $/ \mathrm{m}^{2}$.

标准新风量是测点区域内按照相关规范应当达到 的新风通风量水平. 根据《传染病医院建筑设计规范 (GB50849-2014)》要求，对长庚医院发热门诊各空间 均取标准新风换气次数为 $6 \mathrm{~h}^{-1}$, 计算得到候诊区走 廊、护理站、医生诊室标准新风量分别为 451.8 、 165.6、 $153.0 \mathrm{~m}^{3} / \mathrm{h}$.

利用稀释方程, 计算当室内人数为控制人数上限, 且通风量保持标准新风量时各测点区域二氧化碳的稳 态浓度, 即所述室内二氧化碳浓度理论控制上限:

$C_{\mathrm{u}}=C_{\mathrm{i}}+G / Q$,

其中, $C_{\mathrm{u}}$ 为测点区域室内二氧化碳浓度理论控制上限, $\mathrm{m}^{3} / \mathrm{m}^{3} ; C_{\mathrm{i}}$ 为室外大气中二氧化碳浓度, 即新风中二氧 化碳浓度, 本研究中取 $0.0004 \mathrm{~m}^{3} / \mathrm{m}^{3}$, 即 $400 \mathrm{ppm}(\mathrm{ppm}$, 体积浓度单位, $\left.1 \mathrm{ppm}=10^{-6} \mathrm{~mol} / \mathrm{mol}\right) ; G$ 为室内人员的 二氧化碳总释放速率; $Q$ 为标准新风量, $\mathrm{m}^{3} / \mathrm{h}$. 根据潮气 量 $V_{\mathrm{T}}=500 \mathrm{~mL}$ 、呼吸频率 $R=16 \mathrm{~min}^{-1}$ 、呼出气中二氧 化碳体积浓度 $=4 \%$, 得到人均二氧化碳释放速率 $g=0.0192 \mathrm{~m}^{3} /(\mathrm{h}$ 人). 室内人员的二氧化碳总释放速率 $G$ 
为室内控制人数上限与人均二氧化碳释放速率之积, 即 $G=N_{\mathrm{u}} g, \mathrm{~m}^{3} / \mathrm{h}$. 计算得到长庚医院发热门诊候诊区走 廊、护理站、医生诊室的二氧化碳浓度理论控制上限 分别为 $867 、 864 、 902 \mathrm{ppm}$.

发热门诊聚集性院感风险由人员密度控制范围上 限和二氧化碳浓度理论控制上限这两个指标协同预警. 候诊区走廊实际就诊人员密度通过每日就诊人数换算 得到:

$x^{\prime}=\frac{k X}{T} r \frac{1}{A}$,

其中, $x^{\prime}$ 为计算时刻候诊区实际人员密度, 人 $/ \mathrm{m}^{2} ; k$ 为计 算时刻就诊人数占当日就诊人数比例，长庚医院发热 门诊就诊人数分布规律一般为上午 $(8: 00 \sim 12: 00)$ 和夜间 (18:00 24:00)各占 $1 / 4$, 午后 $(12: 00 \sim 18: 00)$ 占 $1 / 2$, 假设各 时段内就诊人数均匀分布; $X$ 为当日就诊人数, 人; $T$ 为 计算时刻所在时段时间跨度, 对于上午时段为 $4 \mathrm{~h}$, 对于 午后和夜间时段为 $6 \mathrm{~h} ; \mathrm{kX} / T$ 为计算时刻候诊区患者数 量, 人; $r$ 为室内人员数量修正系数, 考虑到长庚医院允 许每位患者携带一位陪同家属, $r$ 的取值范围为 $1 \sim 2$, 本 研究取 1.5 , 即假设发热门诊中半数患者携带有陪同家 属; $A$ 为候诊区域面积, 本研究取 $25.1 \mathrm{~m}^{2}$.

稀释方程基于室内空气均匀混合的假设，因此可 以使用单一测点的数据表征测点空间的整体情况. 发 热门诊环境监测期间的通风方式为自然通风，室内外 温差大, 热压和风压共同作用下通风量大，且疫情期间
就诊患者保持一定间距，同时测点布置于远离医患呼 吸区的位置，所以可认为位于发热门诊候诊区走廊、 护理站和医生诊室的单个测点能代表测点所在空间的 整体通风情况, 即室内空气均匀混合假设合理. 每日二 氧化碳浓度达到最高值时，对应着该空间中聚集性院 感风险当日最高时刻. 将监测时段内每日二氧化碳浓 度最高值与理论限值进行比较, 若超标, 则需进一步观 察分析当日其他时刻二氧化碳浓度的超标情况.

二氧化碳浓度实测值超标，结合室内人员密度估 计值, 对应着两种情况: (1) 室内人员密度过高, 即使自 然通风量能保持达到或超过标准新风量水平，也无法 稀释室内人员呼出的二氧化碳. 此时, 由于密切接触导 致的院感风险增加, 需要控制室内人数, 尤其需要注意 候诊区室内人员密度过高情况. (2) 室内人员密度达标, 但自然通风量未能达到标准新风量水平，需要警惕室 内人源或非人源污染物的累积，尤其需要关注诊疗空 间内新风量不足的情况.

\section{2 院内发热门诊环境控制情况与院感风险 分析}

新冠肺炎疫情暴发以来，北京市委市政府多次召 开会议进行联防联控部署，于2020年1月24日启动突发 公共卫生事件一级响应机制，全力做好疫情防控工作， 保护人民群众的生命安全和身体健康. 1 月 20 日，北京 市首例新冠肺炎患者确诊. 本地病例暴发造成2月初出

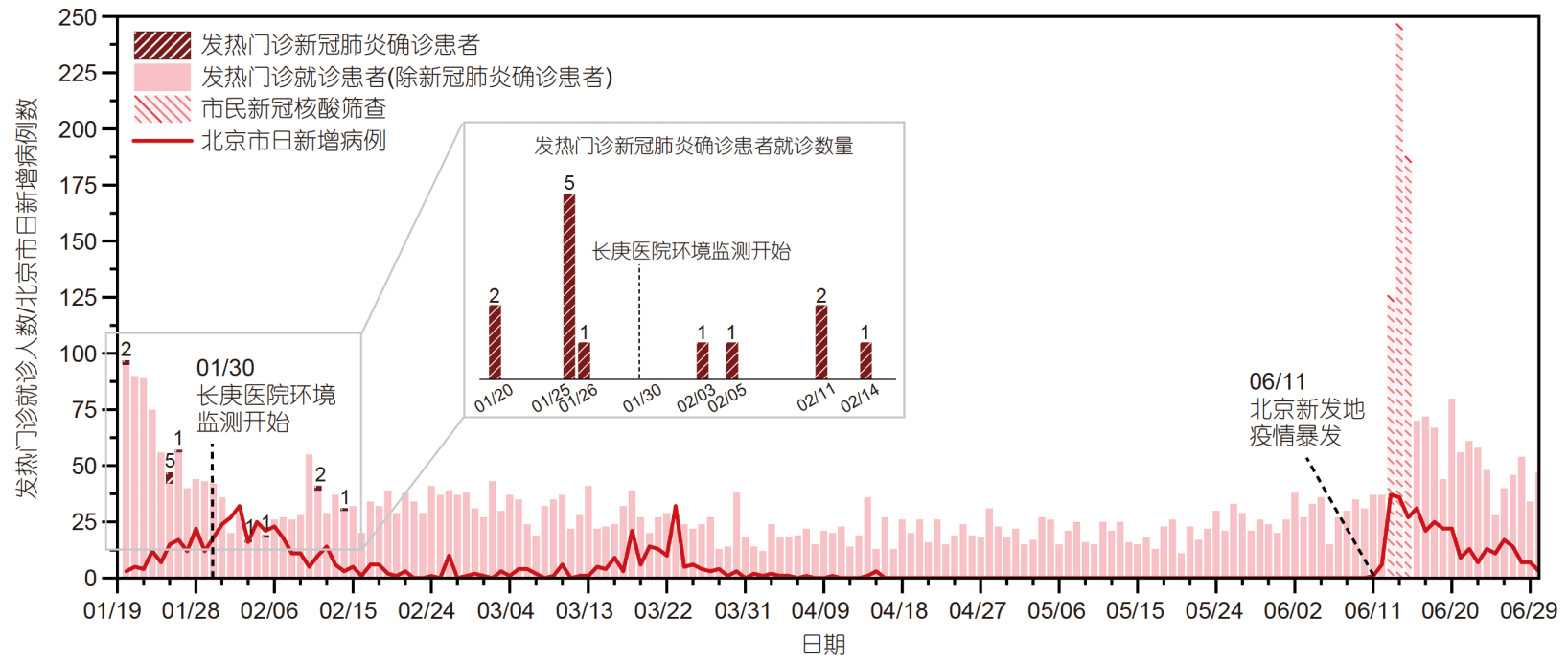

图 3 (网络版彩色)2020年1 6月北京市新冠肺炎病例和清华大学附属北京清华长庚医院发热门诊接诊患者人数统计

Figure 3 (Color online) Number of COVID-19 patients in Beijing, and number of patients attending the fever clinic of Tsinghua University Affiliated Beijing Tsinghua Changgung Hospital from January to June, 2020 
现新增确诊病例高峰. 2月17日之后，长庚医院所在的 昌平区无新增本地确诊病例. 3 月中下旬出现的新增确 诊病例高峰由境外输人造成(图3). 3月底, 北京市新增 确诊病例逐渐趋于 0 . 北京市重大突发公共卫生事件应 急响应级别分别于 4 月 30 日由一级响应下调为二级、 于6月6日由二级响应下调为三级. 6 月 11 日，北京新发 地疫情的首例患者确诊. 6月16日，响应级别重新上调 为二级. 7月 6 日起, 无新发地相关新增本地病例. 参考 北京市疫情发展过程, 选取长庚医院发热门诊2 6月的 环境监测数据, 结合每日就诊人数及人流控制措施, 讨 论分析自然通风条件下发热门诊的环境控制情况及聚 集性院感风险. 由于北京新发地疫情暴发, 在6月13 15 日期间, 长庚医院发热门诊承担大量核酸检测任务, 空 间使用情况具有特异性, 不纳人分析范围; 根据长庚医 院发热门诊患者就诊规律, 后半夜至清晨时段 (00:00 8:00)鲜有患者就诊，且北京市二、三月份室外 温度较低, 此时段室内仅有值班医生时, 可能会将外窗 关闭, 造成室内二氧化碳浓度异常累积, 故该时段亦不 纳人分析范围.

\section{1 发热门诊环境控制情况}

长庚医院发热门诊采用自然通风, 并保持外窗开 启, 保证室内空气流通. 发热门诊护理站测点靠近外窗 (图2(c)). 与医生诊室、候诊区走廊相比, 环境监测时段 内护理站室内温度、相对湿度波动大, 受室外环境影 响程度较大(表3).

\section{2 发热门诊通风实际效果及院感风险分析}

将每日二氧化碳浓度实测最大值与理论限值比较,
结果显示，发热门诊护理站、医生诊室和候诊区走廊 通风稀释情况及人员密度控制情况全部达标(图4). 图4 (a)中散点纵坐标对应发热门诊各测点区域在监测时段 内每日二氧化碳浓度实测最大值. 控制范围上边界为 二氧化碳浓度理论控制限值，候诊区走廊、护理站、 医生诊室的二氧化碳浓度理论控制限值分别为 867 、 $864 、 902 \mathrm{ppm}$ ，标准新风量分别为451.8、165.6、 $153.0 \mathrm{~m}^{3} / \mathrm{h}$. 区域面积未扣除室内设施所占面积, 候诊 区走廊取 $25.1 \mathrm{~m}^{2}$ ，护理站取 $9.2 \mathrm{~m}^{2}$, 医生诊室取 $8.5 \mathrm{~m}^{2}$, 计算得到候诊区走廊、护理站、医生诊室的人员空间 密度范围分别为 $0 \sim 0.44 、 0.22 \sim 0.44 、 0.24 \sim 0.47$ 人 $/ \mathrm{m}^{2}$.

环境监测结果显示，监测期间长庚医院发热门诊 护理站、医生诊室和候诊区走廊室内日二氧化碳浓度 均低于理论限值, 且在新冠肺炎确诊患者停留发热门 诊期间，各区域二氧化碳浓度分别控制在654、711和 609 ppm以下, 自然通风情况良好, 满足传染病医院设 计要求的室内通风稀释水平. 若后续监测中二氧化碳 浓度实测值高于限值, 即出现通风稀释失效的情况, 则 需要增大通风量或降低在室人数限值.

自2020年1月14日以来，长庚医院发热门诊先后接 诊13名新冠肺炎确诊患者. 医院要求医护采取二级防 护, 对环境进行定时消杀, 并采用自然通风确保通风稀 释情况良好. 这 3 种感控途径共同保证了医护零感染、 患者之间零交叉感染, 在一定程度上可以证明本研究 基于室内人员密度和二氧化碳浓度限值的发热门诊院 感风险实时预警方法的可行性与合理性.

需要指出的是, 该方法在长庚医院发热门诊的实 际运用中也存在一些局限性. 第一, 在环境监测空间通 风量充足、室内源分布均匀且测点远离源时, 可认为

\section{表 3 发热门诊监测期间环境参数}

Table 3 Environmental parameters during fever clinic monitoring

\begin{tabular}{|c|c|c|c|c|}
\hline \multicolumn{2}{|c|}{ 监测参数 } & 护理站 & 医生诊室 & 候诊区走廊 \\
\hline \multirow{2}{*}{ 温度 $\left({ }^{\circ} \mathrm{C}\right)$} & 中位数(最小值 最大值) & $18.0(5.9 \sim 34.7)$ & $16.3(5.2 \sim 29.6)$ & $15.2(4.7 \sim 22.9)$ \\
\hline & 平均值士标准差 & $18.2 \pm 4.7$ & $16.4 \pm 3.3$ & $15.2 \pm 2.8$ \\
\hline \multirow{2}{*}{ 相对湿度(\%) } & 中位数(最小值 最大值) & $14.7(1.8 \sim 60.7)$ & $17.9(3.8 \sim 50.7)$ & $20.3(6.9 \sim 49.9)$ \\
\hline & 平均值 \pm 标准差 & $17.9 \pm 11.3$ & $19.3 \pm 8.1$ & $22.1 \pm 8.5$ \\
\hline \multirow{2}{*}{ 二氧化碳浓度(ppm) } & 中位数(最小值 最大值) & $507(409 \sim 665)$ & 464(394 985) & $437(379 \sim 785)$ \\
\hline & 平均值土标准差 & $512 \pm 46$ & $473 \pm 45$ & $447 \pm 40$ \\
\hline \multirow{2}{*}{$\mathrm{PM}_{2.5}$ 质量浓度 $\left(\mu \mathrm{g} / \mathrm{m}^{3}\right)$} & 中位数(最小值 最大值) & $23(1 \sim 785)$ & $29(1 \sim 258)$ & $31(3 \sim 200)$ \\
\hline & 平均值土标准差 & $29.9 \pm 24.6$ & $36.8 \pm 35.5$ & $37.5 \pm 31.9$ \\
\hline
\end{tabular}



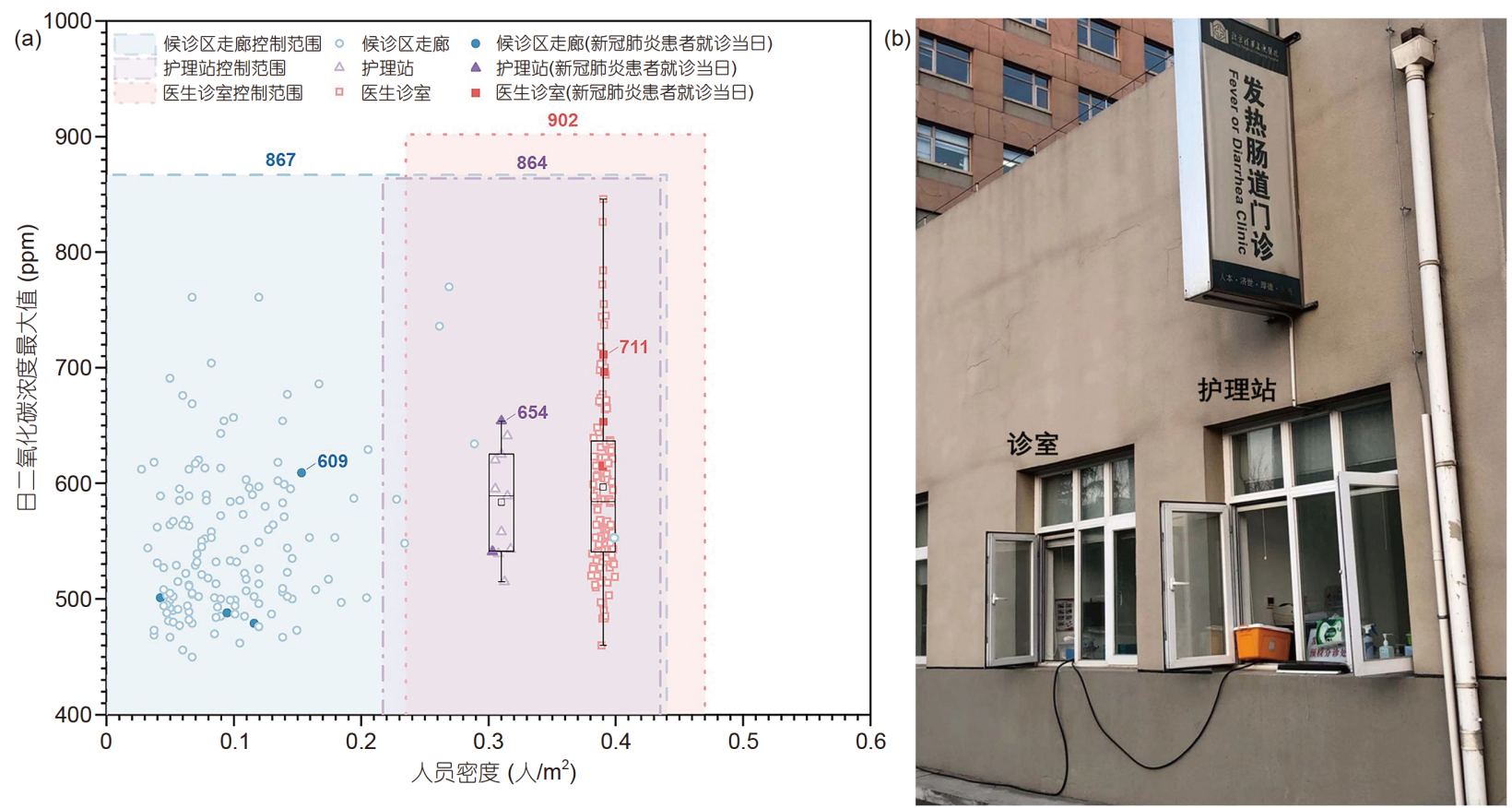

图 4 (网络版彩色)长庚医院发热门诊通风控制情况. (a) 发热门诊各测点空间二氧化碳浓度限值范围及监测时段日二氧化碳浓度实测最大值; (b) 发热门诊就诊空间开窗情况

Figure 4 (Color online) Ventilation performance at the fever clinic in Tsinghua University Affiliated Beijing Tsinghua Changgung Hospital. (a) Maximum allowed range of $\mathrm{CO}_{2}$ concentration and occupant density, as well as the measured daily maximum $\mathrm{CO}_{2}$ concentration during monitoring; (b) window opening situation at the fever clinic

监测空间内空气均匀混合且空间内单一测点能表征整 个空间的通风情况. 本研究中的发热门诊地处北京, 疫 情期间自然通风条件良好，能够满足室内空气混合均 匀假设. 若应用于其他自然通风环境中, 需注意室内源 的分布情况, 判断监测空间内空气是否混合均匀; 应用 于机械通风场合时，必须计算监测空间通风量是否充 足并关注室内源，证明监测空间满足空气均匀混合假 设. 第二, 就诊人数数据时间精度较低, 各时段的实际 接诊人数未知, 需接受各时段占每日接诊人数比例固 定, 且采用就诊人数在各时段中均匀分布的假设, 进行 对候诊区走廊实际人员密度的反算. 第三, 环境监测参 数及室内人数信息不完整. 环境监测数据在监测时段 内因设备断电等原因有所缺失，而已知确定的室内人 数信息仅包括就诊患者人数，未包括陪同就诊的家属 人数，在反推候诊区走廊实际人员密度时利用放大系 数进行修正. 第四, 室外二氧化碳浓度受交通、季节、 天气等因素影响而波动, 但长庚医院外无二氧化碳工 业排放源，外部公路、停车场等二氧化碳散发源距离 发热门诊大于 $100 \mathrm{~m}$, 且监测时段院内无人为取暖活 动, 因此本研究忽略了室外源的影响, 认为室外新风中
二氧化碳浓度变化不大，对控制限值的影响可忽略不 计. 若应用于其他医院, 需注意院外二氧化碳散发源对 室内二氧化碳浓度基准值的影响.

\section{3 结论与建议}

新冠肺炎疫情暴发以来，综合医院发热门诊以及 新冠肺炎定点医院接收了大量新冠肺炎患者和疑似患 者, 医院环境承载力面临挑战，需要通过控制就诊人 数、规划人员流线、强化通风稀释、完善医护防护措 施、定时消杀环境物表等院感防控措施，争取将院内 医护人员和未感染患者的院感风险降到最低. 针对医 院内可能存在的气溶胶传播, 强化通风稀释对切断传 播途径尤为重要, 亟须寻求普适、实时、便捷的环境 监测方法表征医院环境的感染风险和通风稀释水平, 并以量化指标对院感风险进行预警.

本研究以清华大学附属北京清华长庚医院为例, 提出了一种基于环境参数监测情况实时预警院内聚集 性院感风险的方法, 以室内二氧化碳浓度限值和人员 密度为核心指标划定环境控制范围，可根据二氧化碳 浓度实测值快速识别综合医院发热门诊内出现高院感 
风险的区域，进而有针对性地采取干预措施保护医 患、改善医院环境. 根据发热门诊候诊区走廊、护理 站和医生诊室在室人数限值及规定新风量，计算出上 述区域内二氧化碳浓度理论上限值分别为 867 、864和 $902 \mathrm{ppm}$ ，环境监测时二氧化碳浓度分别控制在609、 654和711 ppm以下。个体防护是医生近距离接触患者 时降低暴露风险最重要的一道防线. 长庚医院环境监 测期间, 发热门诊医生均采取二级防护, 未有医患间感
染发生. 此外, 监测阶段内发热门诊各区域二氧化碳浓 度低于理论限值, 自然通风稀释有效, 聚集性院感风险 较低. 自2020年1月30日环境监测开始，发热门诊共接 诊5名新冠肺炎确诊患者，就诊患者佩戴口罩，室内二 氧化碳浓度低于理论限值并保持在609 711 ppm以下， 未发生患者间感染，这说明我们提出的环境控制指标 与院感预警方法具有合理性，可供同类型综合医院发 热门诊设计与改造参考.

\section{参考文献}

1 Chu D K, Akl E A, Duda S, et al. Physical distancing, face masks, and eye protection to prevent person-to-person transmission of SARS-CoV-2 and COVID-19: A systematic review and meta-analysis. Lancet, 2020, 395: 1973-1987

2 Liu L, Li Y, Nielsen P V, et al. Short-range airborne transmission of expiratory droplets between two people. Indoor Air, 2017, 27: 452-462

3 Epidemiology Working Group for NCIP Epidemic Response, Chinese Center for Disease Control and Prevention. The epidemiological characteristics of an outbreak of 2019 novel coronavirus diseases (COVID-19) in China (in Chinese). Chin J Epidemiol, 2020, 41: 145-151 [中国 疾病预防控制中心新型冠状病毒肺炎应急响应计值流行病学组. 新型冠状病毒肺炎流行病学特征分析. 中华流行病学杂志, 2020, 41: 145151]

4 Chang D, Lin M, Wei L, et al. Epidemiologic and clinical characteristics of novel coronavirus infections involving 13 patients outside Wuhan, China. JAMA, 2020, 323: 1092-1093

5 Han J B, Jia Z K, Yu L H, et al. Heating ventilation and air conditioning design for Beijing Tsinghua Changgung Hospital (in Chinese). HV\&AC, 2016, 46: 70-75 [韩佳宝, 贾昭凯, 于丽华, 等. 北京清华长庚医院暖通空调设计. 暖通空调, 2016, 46: 70-75]

6 van Doremalen N, Bushmaker T, Morris D H, et al. Aerosol and surface stability of SARS-CoV-2 as compared with SARS-CoV-1. N Engl J Med, 2020, 382: 1564-1567

7 Chaudhuri S, Basu S, Kabi P, et al. Modeling the role of respiratory droplets in Covid-19 type pandemics. Phys Fluids, 2020, 32: 063309

8 Mirhoseini S H, Nikaeen M, Khanahmd H, et al. Monitoring of airborne bacteria and aerosols in different wards of hospitals-Particle counting usefulness in investigation of airborne bacteria. Ann Agric Environ Med, 2015, 22: 670-673

9 Batterman S. Review and extension of $\mathrm{CO}_{2}$-based methods to determine ventilation rates with application to school classrooms. Int J Env Res Pub He, 2017, 14: 1-22 


\title{
Environmental monitoring and infection control of fever clinics in general hospitals during COVID-19 pandemic
}

\author{
Yiran $\mathrm{Lu}^{1,2 \dagger}$, Yifan $\mathrm{Li}^{1,2 \dagger}$, Minggui $\mathrm{Lin}^{3^{*}}$, Li Liu ${ }^{1,2^{*}}$, Borong $\operatorname{Lin}^{1,2}$, Hao Zhou ${ }^{1,2}$, Jinlan $\operatorname{Lin}^{3} \&$ \\ $\mathrm{Huji} \mathrm{Xu}^{3}$ \\ ${ }^{1}$ School of Architecture, Tsinghua University, Beijing 100084, China; \\ 2 Laboratory of Eco-Planning \& Green Building, Ministry of Education, Tsinghua University, Beijing 100084, China; \\ ${ }^{3}$ Department of Infection, Tsinghua University Affiliated Beijing Tsinghua Changgung Hospital, Beijing 102218, China \\ $\uparrow$ Equally contributed to this work \\ *Corresponding authors, E-mail: linminggui309301@126.com; liuli_archi@tsinghua.edu.cn
}

The early stage of the COVID-19 epidemic happened to be the flu season. Since some symptoms of influenza and COVID19 are similar, symptomatic patients flocked to fever clinics and emergency departments. Meanwhile, asymptomatic COVID-19 patients attending other departments in general hospitals made things worse. Lack of knowledge of the pathogen, absence of awareness and short of personal protective equipment all posed threat to healthcare workers as well as other patients. As SARS-CoV-2 can be spread via droplets, direct contacts and potentially aerosols, the indoor air environment of hospitals, especially fever clinics, must have strict measures to prevent hospital-acquired infection.

Thirty-two sensors were deployed in the Tsinghua University Affiliated Beijing Tsinghua Changgung Hospital (mentioned as Changgung Hospital hereinafter) from January 30, 2020, in order to monitor high-resolution real-time indoor environmental parameters at its fever clinic, isolation wards and other departments. One sensor monitors and records $\mathrm{CO}_{2}$ concentration, $\mathrm{PM}_{2.5}$ mass concentration, relative humidity, temperature and illuminance every 5 minutes. Six sensors were located at the fever clinic, where all patients with fever and/or other COVID-19 related symptoms firstly attended after arriving at the hospital. The clinic has two parts, one for diagnosis and the other for quarantine. Three sensors were placed in doctor's office, nursing station and waiting area in the diagnosis part, respectively. Natural ventilation was chosen to dilute the environment, as the flowrate of outdoor airflow was abundant in Beijing's winter.

Atmospheric $\mathrm{CO}_{2}$ concentration surrounding Changgung Hospital was stable, and the rise of indoor $\mathrm{CO}_{2}$ concentration was caused by human exhalation. During this pandemic, $\mathrm{CO}_{2}$ concentration can be regarded as an indicator of room ventilation condition and hospital congestion, if all the people in hospital were regarded as potential infector of SARS$\mathrm{CoV}-2$. According to the usage pattern of the fever clinic, the maximum number of patients in each functional area was set as 4 for doctor's office, 4 for nursing station and 11 for waiting area. According to the ventilation regulation of infectious disease hospital, the air change rate at fever clinics should be at least $6 \mathrm{~h}^{-1}$. In addition, the outdoor $\mathrm{CO}_{2}$ concentration was assumed to be $400 \mathrm{ppm}$. Based on these conditions, the upper limits of indoor $\mathrm{CO}_{2}$ concentration were 902,864 and 867 ppm for doctor's office, nursing station and waiting area at the Changgung Hospital's fever clinic, respectively. Indoor $\mathrm{CO}_{2}$ concentration exceeding these thresholds stands for poor ventilation or overcrowds. Fortunately, this didn't happen during the monitoring period and indoor $\mathrm{CO}_{2}$ concentration didn't exceed 609-711 ppm. In another word, natural ventilation was sufficient and effective in this specific case at the Changgung Hospital's fever clinic. Moreover, together with environment disinfection and personal protective measures, good ventilation condition led to no COVID-19 hospitalacquired infection.

To conclude, this article introduced a real-time environmental monitoring campaign at the Changgung Hospital's fever clinic. Similar methodology can help assess ventilation conditions and risk of hospital-acquired infection at the fever clinic during and after COVID-19 pandemic. Once indoor $\mathrm{CO}_{2}$ concentration exceeds the set thresholds, areas with high infection risk can be identified rapidly and timely, so that prevention measures can be taken in time.

COVID-19, hospital environment, monitoring and warning, infection prevention and control doi: 10.1360/TB-2020-0969 